\title{
SIII-1b \\ Design and manufacturing of an unmanned aerial vehicle with air quality control system
}

\section{Subtitle}

\author{
H. Smajic ${ }^{1}$, T. Duspara ${ }^{2}$
}

\begin{abstract}
Within the scope of this project, a fully functional model of an unmanned aerial vehicle with an air control system was developed and implemented as a prototype. By using the mobile measuring stations, it is feasible to measure the air quality in any part of the city, independent of the measuring altitude.
\end{abstract}

\section{Introduction}

The development of unmanned aerial vehicles in the very beginning was secretive, mostly because they were commonly used for military purposes. Today in the 21 st century, they are in all segments of life, from military, civilian to recreational $[4,5]$. Their landing and take-off is possible from different surfaces, from grassy, sandy to paved surfaces. Unmanned aerial vehicles are aircrafts capable of performing continuous flight without a pilot [1,2]. The use of drones, especially for civilian purposes, has grown exponentially, in terms of both number, size and weight, as well as the increasing number of applications.

\subsection{Classification of unmanned aerial vehicles}

In reality, "Drone" refers to a multi-jet aircraft. These vehicles are always unmanned and traditionally consist of three, four, six or eight arms. Each arm features a high performance engine and propeller, allowing the aircraft to lift considerable weight and maneuver around tight spaces. According to the basic division by number of rotors, aircraft are divided into Tricopter (three rotors), Quadcopter (four rotors), Hexacopter (six rotors), Octacopter (eight rotors) etc.

\section{Overview of main components of an UAV}

To design the unmanned aerial vehicle, it is necessary to specify six main components of every unmanned aerial vehicle.

- Drone Body

- Drive train (Electromotor with propellers)

- Power train (Battery)

- Power distribution system

- Communication system (Radio communication)

- Control system (Flight Computer)

Depending on the purpose of drone, there is one additional subsystem

Sensor system

\footnotetext{
1 Prof. Dr. Ing. Hasan Smajic, TH Koln/Faculty of Vehicle Systems and Production, Institute, Cologne, Germany 2 BSc Eng. Toni Duspara, TH Koln/Faculty of Vehicle Systems and Production, Institute, Cologne, Germany
} 
For any type of unmanned aerial vehicle, the all major components must be included. They are not related to the number of rotors or the purpose of the drone. The load or device carrying the drone depends on the purpose of the drone. It constitutes the sixth subsystem mentioned in previous chapter. In this case, it is necessary to design a drone capable of carrying a sensor that can interrogate air quality data and parameters. Additional sensor represents the seventh subsystem.

\subsection{Selection of components}

\subsubsection{Body parameters}

From the requirements set out, it was clear that a "quadcopter" must be built as an unmanned aircraft with four rotors. The name itself tells us that the aircraft consists of four electric motors which generate the pushing force and lift the aircraft into the air. The material used for the construction of the drone body is PLA plastic with $20 \%$ filling. This type of plastic is suitable for the construction of an airplane because it can be produced with rapid prototyping machines (3D printers). To start designing the drone body, it is essential to model and design the frame itself. The diameter of a complete assembly will be about $450 \mathrm{~mm}$. From diameter size, the length of the arms will be derived. The weight of the aircraft should not exceed $1 \mathrm{~kg}$. In this way, the flight time will be extended.

\subsubsection{Drive train}

To select a suitable drive train assembly consisting of propeller, brushless motor and ESC, suitable propellers and their length must be defined. According to the frame dimensions of $450 \mathrm{~mm}$ diameter 10" (approx. 250mm) propellers will be eligibile for this prototype. Four propellers have to be purchased, two of as work clockwise and two counterclockwise. A proper electric motor should be selected for the next step. A brushless DC electric motor is most often used for the design and construction of unmanned aircraft. The motor types are declared with KV unit. KV stands for the number of revolutions minute per one volt. Since the aircraft uses a LiPo battery with a nominal voltage of about 11 volts, a suitable motor should be equipped with a unit of about $1000-1800 \mathrm{KV}$. The last component of the drive train system is an ESC speed controller. ESC controllers are quite durable. Using a $30 \mathrm{amp}$ controller for the drivetrain, should be enough to control the speed of electric motors smoothly.

\subsubsection{Power source (battery)}

The battery has a very importance for airplanes. It drives the power train together with all other components within a drone. A three-cell LiPo battery is most often chosen for aircraft design. The battery types generate a voltage of $11.1 \mathrm{~V}(3.7 \mathrm{~V}$ per cell). The capacity of a battery has a direct effect on flight time. The greater the capacity, the longer the flight time. It is also important that a larger capacity indicates a heavier battery. The capacity of a battery is selected according to the size of the drone body. For a drone body size of $450 \mathrm{~mm}$ the suitable battery is a 3-cell LiPo battery with 2500 to 4000 mAh. Accordingly, the most suitable battery is a 3-cell LiPo battery with $2700 \mathrm{mAh}$ and XT60 connection.

\subsubsection{Transmitter and receiver}

Radio connection assembly consists of two main parts: transmitter and receiver. Every unmanned vehicle system can use the same type of a transmitter and receiver. The transmitter chosen for this project is a FS-i6 six channel regulator. Four of the channels are controlled by 
joysticks and the other two are free for programing. The last component that comes with the transmitter is a receiver. Receiver is attached to the drone body. It is connected directly to the flight computer, transmitting the sensor signals via radio connection.

\subsubsection{Flight computer}

For the data processing in the air a flight controller had to be found first. The controller KK2.1.5 has an LCD display, which makes programming and calibration of the processes much easier. Particular advantages are provided by pre-installed flight types and drone profiles. These features make it easier to set the type of aircraft, check the direction of rotation of all motors, route the current flow through the ESC-s and perform the calibration.

\subsubsection{Power distribution}

Power distribution to all of components is usually achieved through custom PCB boards. The PCB boards are directing the current flow to all necessary parts using a parallel connection scheme. The board itself has sockets for all four ESC controllers (and can hold up to six units). A big advantage of this board is the integrated connections for other devices that have to operate at a different voltage. A nominal voltage of $5 \mathrm{~V}$ is required to supply the sensor system, consisting of a microcontroller and detectors. Since the LiPo battery supplies a DC voltage of $11.1 \mathrm{~V}$, a direct connection to the sensor system is not possible. By employing the distribution board, a $5 \mathrm{~V}$ power source is available to supply the entire sensor system.

\subsection{Components for drone design}

Following the design of the drone concept and the specification and dimensioning of all necessary units, the list of components can be summarized in Table 1.

\begin{tabular}{|l|l|r|r|r|}
\hline \multicolumn{5}{|c|}{ PARTS FOR DRONE CONSTRUCTION } \\
\hline \multicolumn{1}{|c|}{ Name } & \multicolumn{1}{|c|}{ Mark } & Qt & Price/pc (USD) & Price (USD) \\
\hline ESC & XXD HW30A & 4 & 2,99 & 11,96 \\
\hline DC Motor & A2212 & 4 & 8,99 & 35,96 \\
\hline Flight computer & KK2.1.5 & 1 & 14,90 & 14,90 \\
\hline LiPo Charger & IMAX B6 & 1 & 27,02 & 27,02 \\
\hline Propellers & $104510 \times 4.5$ & 1 & 9,26 & 9,26 \\
\hline LiPo Battery & 3S 2700mAh & 1 & 19,17 & 19,17 \\
\hline Communication & FS-i6X 10CH & 1 & 54,70 & 54,70 \\
\hline PDB & FDB XT60 & 1 & 2,51 & 2,51 \\
\hline Battery connector & XT60 & 1 & 0,81 & 0,81 \\
\hline Microcontroler & ESP8226 & 1 & 2,79 & 2,79 \\
\hline Particle sensor & PMS5003 & & 30,5 & 30,5 \\
\hline SHIPPING & \multicolumn{3}{|c|}{$\$ 24,02$} \\
\hline ALTOGETHER
\end{tabular}

Table 1: Parts for drone construction 


\section{Modelling and design of the aircraft}

The modelling and construction of a quadcopter consists of the design of the drone body itself. The body consists of a central part and arms that support the rotors. They are the main components of any UAV or drone. To avoid collisions between the propellers and to achieve a compact design, it has already been specified that the diagonal distance between the rotors should be between 400 and $500 \mathrm{~mm}$. The approximate dimensional area of an aircraft is shown in below.

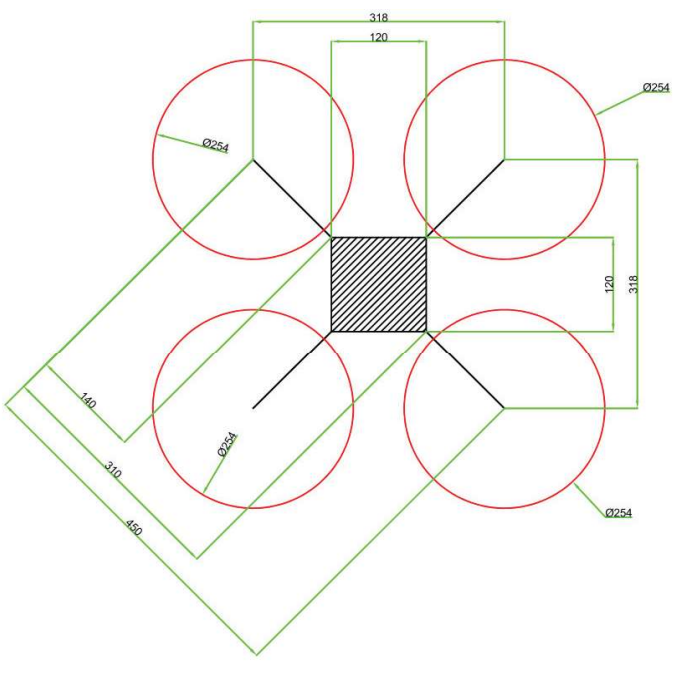

Figure 1: a) Dimensional area

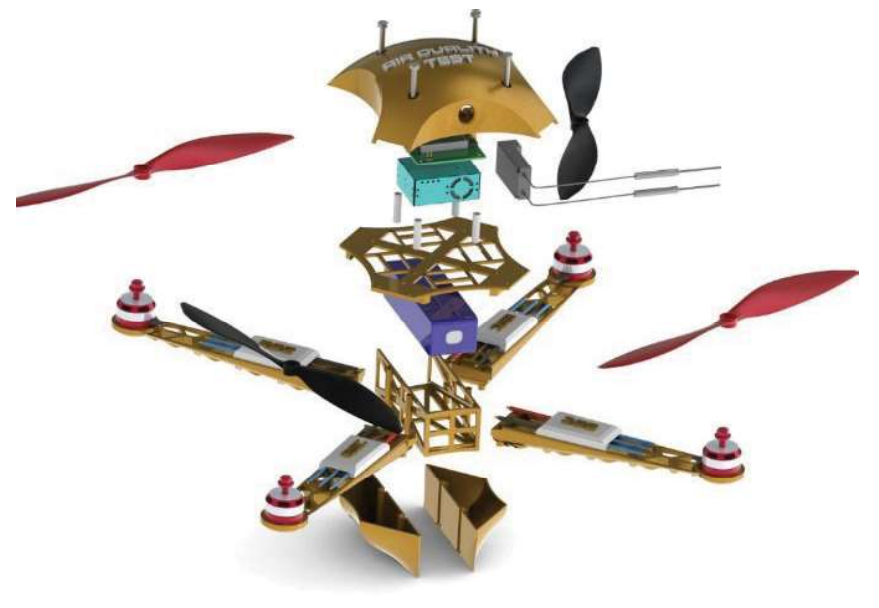

b) Exploded drone body with all components

First, it is necessary to determine the dimensions of the central part of an aircraft. The main task of the central part is to carry the electronic components. The part itself should have brackets for battery, flight computer, sensor system, radio receiver etc. The approximate dimensional area for the central part is $120 \times 120 \mathrm{~mm}$. According to this it is specified that the arms of an airplane should be between 140 and $200 \mathrm{~mm}$ long. During the construction it is crucial to pay attention to the mass of the parts. To reduce the mass of certain parts, it is recommended to model the parts so that they are perforated. By strategically piercing the holes inside the body panels, the mass is reduced in a way that does not compromise the integrity of the structure. Another advantage of reducing mass and piercing holes is that air can flow through the arms. This helps to get more benefit from the propulsion assembly and the propellers themselves. All components are connected by nuts and bolts. The dimensions of the arm model are $190 \times 40 \times 20 \mathrm{~mm}$, which fits perfectly within the given dimensional range. The central part of this airplane, which is connected with four arms and to which all other parts are attached, should fit into a dimensional range of about $120 \times 120 \mathrm{~mm}$. When attaching the components to the central part, the center of mass should not be forgotten.

For an aircraft to have a stable flight, it is essential that the centre of mass is in the middle of the aircraft. To avoid calculating the center of mass for each individual component and later having to calculate the positions of the components, the easiest way is to simply maintain the symmetry of an airplane [3]. This is achieved by arranging the components in layers. Accordingly, the battery that powers the entire system should be placed under the central part and the flight computer and sensor systems should above the central part. The positioning of the components on the central part is done by model mounts. To arrange the components in layers, we will design spacers. The main task of the spacers is to lift the flight computer above the central part so that the senosr system can be placed in between. The final assembly with all components weighs about $750 \mathrm{~g}$. Once the drone body has been designed, it is recommended to create the housing. The main function of the housing is to protect the electronic components. The modelling of the housing contributes to the aesthetic appearance of an aircraft (Figure 1b). The enclosure consists of four parts: upper cap, lower left cap, lower right cap, ESC caps. 


\section{Air quality measuring system}

\subsection{Introduction}

After the construction of the drone itself, or the drive unit of the project, it is necessary to design and configure the measurement subsystem. Before the plan itself is designed, it is essential to describe what makes up the measurement system and the need behind the construction of this project. The unit is called the Air Quality Index and was developed by the US Environmental Protection Agency. Based on the value of the AQ index, it is possible to extrapolate the level of air quality and the likely health risks to which the population could be exposed after a number of hours or days of exposure to the air itself. The data from the automatic measurement units are obtained hourly from the relevant government institution and published on the official website. Fine, suspended particles, PM2.5, are mainly the product of various combustion processes. Because of their small size, they can penetrate deep into the lungs and reach most, if not all, of the internal organs via the bloodstream, so they pose a far greater health risk than coarse suspended particles.

\subsection{Sensor Detection}

Since the desired measurement result represents the air quality index, or rather, the concentration of airborne particles, it is necessary to select a sensor with the ability to display the desired measurements. With that in mind, the PMS5003 sensor was selected for this project The PMS5003 is a type of digital and universal sensors which measures airborne particles in the air and displays the readings in digital form. The measurement system of this sensor is based on laser scattering technology. A laser diode within the sensor irradiates particles coming in to the sensor. A receiver across the diode measures the amount of scattered light. The microprocessor inside the sensor allows us to calculate the nominal diameter of the particles and the number of particles using the MIE theory.

The sensor displays values of PM1.0, PM2.5, and PM10 concentrations in $\mu \mathrm{g} / \mathrm{m}^{3}$ as output. There are two output options with the sensor: a passive output and an active output. The default output option while powering on the device is active output mode. If the changes in concentration values are very low, the sensor will work in stable mode that will output data every 2,3 seconds. If the sensor detects rapid and increased differences in concentration, it will automatically switch to fast mode with outputs data every $200 \sim 800 \mathrm{~ms}$. The larger the concentration of the particles, the faster will the sensor respond. Response time also depends on the upload speed to the online server.

\subsection{Control system}

To fully utilize the PMS5003 sensor, it must be connected to a microcomputer that processes the sensor data and displays it to the user. Since the drone is a mobile unit, the microcomputer must have means for wireless communication. Due to the fact that the drone itself uses a radio link for communication between the transmitter and receiver, and due to the fact that Bluetooth has a short range, it is best to use Wi-Fi communication. One of the important features when selecting the appropriate control system is to secure the power source. Sensors and microcomputers usually work with low voltage (5V). The NODEMCU microcomputer was adopted because it has wireless communication and a direct internal power supply. The microcomputer contains an integrated ESP2668 module for wireless communication over Wi-Fi networks. A mobile device (cell phone) with $\mathrm{Wi}-\mathrm{Fi}$ is used to connect the microcontroller to the Internet and display the measurement results on either an online server or a local server. 


\subsection{Sensor system wiring}

When approaching the wiring of sensor systems, the first step is to check the pin layout and pin function on each device (microcontroller and PMS5003 sensor). The complete wiring diagram between the sensor system components is shown in Figure 2. All devices in the system are wired to the same power source (LiPo battery). The flight computer is powered by the ESC-s, while other components receive their power from a power distribution unit connected to the battery.

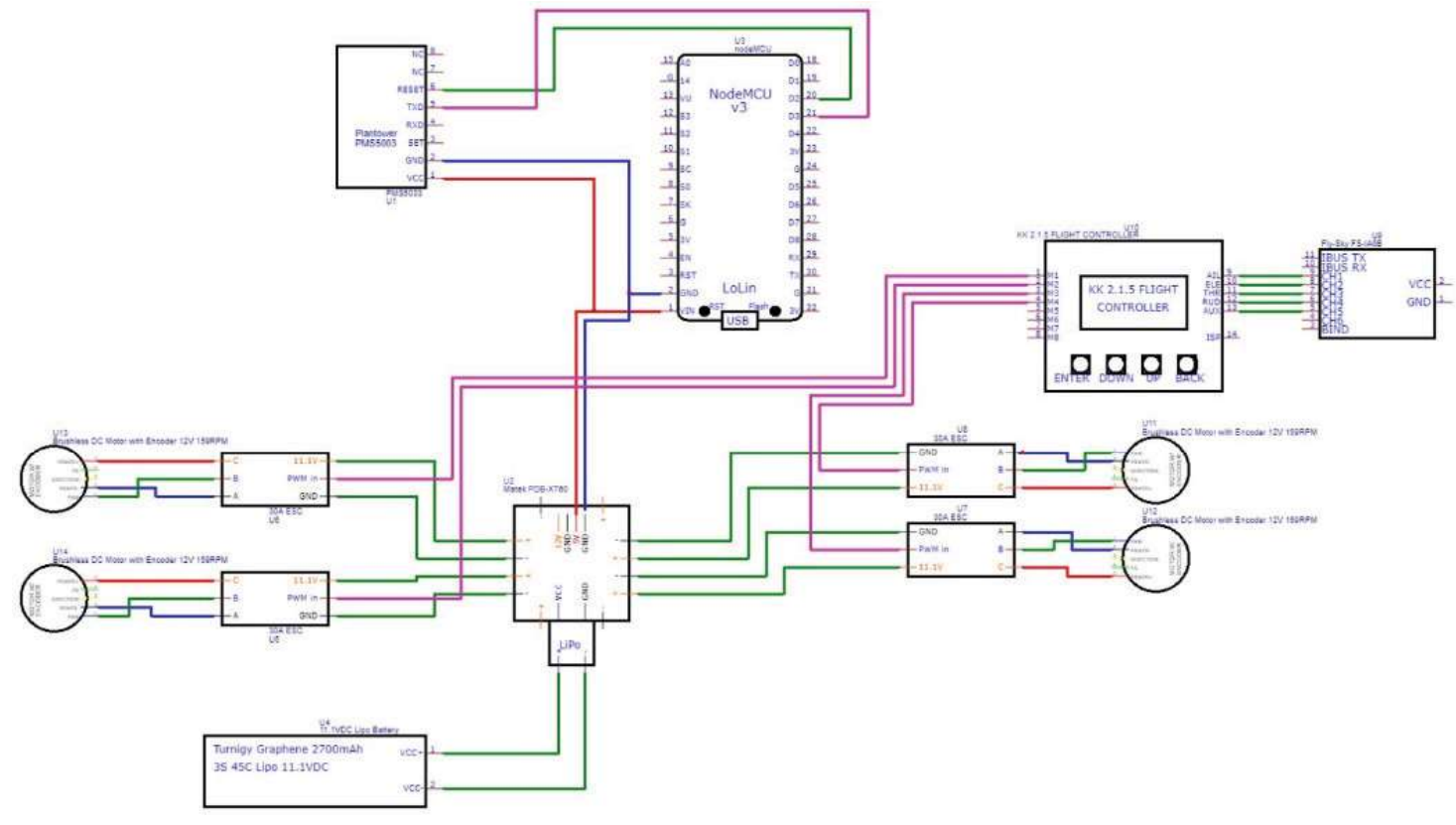

Figure 2 - Drone system wiring

\subsection{Measuring system automation}

As already mentioned, the development of control system software is done with the integrated development environment Arduino. Additional software still needs to be developed. This software creates a server on a personal computer that stores measurement data. Py-thon programming language was used as the development platform. In order to be able to view the measurement data at any time and any place, the data is automatically uploaded to an online server. Using a Wi-Fi adapter integrated in a microcontroller, the aircraft can be connected to the Internet via a mobile device (personal hotspot). Once the connection between the microcontroller and the mobile device is established and later when the aircraft is connected to the Internet, the data is automatically uploaded to an online server. In this case, the computer connected to the Internet will be the local server where the data is stored.

\subsubsection{Server establishment with python}

When the main parameters (IP address, connection number, etc.) have been set, the software goes into a cycle. In this loop, the server initially waits for the connection of the client. Upon establishing the connection, the server outputs the IP address of the client and starts receiving data from the microcontroller. During their connection, the server and client constantly send messages to each other to ensure their connection. If the server detects that no data is coming in, the loop stops and the software tries to connect again. When both devices are turned on and connected to the Internet, the connection is automatically activated and data is exchanged. 


\subsubsection{Software development for sending and receiving measurement data}

The second part of automation of measurement systems is the development of software that receives data from the sensor and transmits it to the server. This software has two purposes. The first is to establish the connection between the sensor and the microcontroller to obtain measurement results. The second is sending these results to the server. The controller constantly receives a cluster of data (Figure 5).

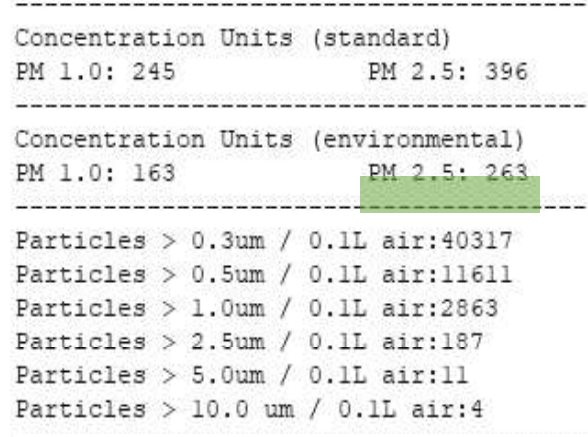

PM 10: 406

PM 10: 270

Figure 3: Python code output

\subsubsection{Results preview and printout}

When measurement data has been successfully exchanged between server and client, it is necessary to print them. The code automatically connects the server to Google Cloud and stores the measurement data in an Excel spreadsheet. The measurement data are automatically uploaded online and can be accessed from anywhere.

\section{Conclusion}

Within the framework of this project, a system for air quality control with unmanned aircraft was successfully designed and implemented. The design and construction with all its components was the first step towards production. The aircraft measures approximately $450 \times 450 \mathrm{~mm}$ and is made of PLA plastic using 3D printing technology. For the propulsion system 1400KV brushless DC motors with 10" propellers and 30A ESC-s were applied. The control unit consists of the flight computer KK2.1.5, which is connected to a FS-i6 transmitter and receiver. Power source for the whole system was a three-cell LiPo battery with $2700 \mathrm{mAh}$ capacity. The entire aircraft with all its components weighs about $750 \mathrm{~g}$ and due to its characteristics falls into the "mini" type of a drone. The sensor system includes the microcontroller ESP8226 with built-in Wi-Fi module and the air particle sensor PMS5003. The results are immediately stored on a server after the measurement. The first is a local server (operator's personal computer) and the second is an online Google server. Finally, the data are saved in an Excel table.

\section{References}

[1] Cinnamon, I., Kadri, R. and Tepper, F., 2016. DIY Drones For The Evil Genius. New York: McGraw-Hill Education TAB

[2] Yong, Z., 2016. Digital universal particle concentration sensor (PMS5003 Series data manual), 2016 product data manual of PLANTOWER

[3] D. Norris, Build your own quadcopter. New York: McGraw-Hill Education, 20144.

[4] A. Baum, Verkehrsträgerübergreifende Messung von Luftschadstoffen mit Dronen, 1. Tagung des Expertennetzwerks, 14.06.2018, BMVI, Berlin

[5] https://www.drohnen.de/category/drohnen-einsaetze/page/16 